Published in final edited form as:

Prostaglandins Leukot Essent Fatty Acids. 2014 September ; 91(3): 87-96. doi:10.1016/j.plefa. 2014.04.001.

\title{
Is the omega-3 index a valid marker of intestinal membrane phospholipid EPA+DHA content?
}

\author{
Eric A. Gurzell ${ }^{1}$, Jason Wiesinger ${ }^{1}$, Christina Morkam ${ }^{1}$, Sophia Hemmrich ${ }^{1}$, William S. \\ Harris $^{3}$, and Jenifer I. Fenton ${ }^{1,2,{ }^{*}}$ \\ ${ }^{1}$ Department of Food Science and Human Nutrition, Michigan State University, East Lansing, MI \\ ${ }^{2}$ College of Osteopathic Medicine, East Lansing, MI \\ ${ }^{3}$ Sanford School of Medicine, The University of South Dakota, Sioux Falls, SD
}

\section{Abstract}

Despite numerous studies investigating n-3 long chain polyunsaturated fatty acid (LCPUFA) supplementation and inflammatory bowel diseases (IBD), the extent to which dietary n-3 LCPUFAs incorporate in gastrointestinal (GI) tissues and correlate to the omega-3 index is unknown. In this study, mice were fed three diets with increasing percent of energy (\%en) derived from eicosapentaenoic acid (EPA) + docosahexaenoic acid (DHA). Dietary levels reflected recommended intakes of fish/fish oil by the American Heart Association. We analyzed the FA composition of phospholipids extracted from red blood cells (RBCs), plasma, and GI tissues. We observed that the 0.1\% en EPA+DHA diet was sufficient to significantly increase the omega-3 index (RBC EPA+DHA) after 5 week feeding. The baseline EPA levels were $0.2-0.6 \%$ across all tissues increasing to $1.6-4.3 \%$ in the highest EPA+DHA diet; these changes resulted in absolute increases of $1.4-3.9 \%$ EPA across tissues. The baseline DHA levels were $2.2-5.9 \%$ across all tissues increasing to $5.8-10.5 \%$ in the highest EPA+DHA diet; these changes resulted in absolute increases of $3.2-5.7 \%$ DHA across tissues. These increases in EPA and DHA across all tissues resulted in strong $(r>0.91)$ and significant $(\mathrm{p}<0.001)$ linear correlations between the omega-3 index and plasma/GI tissue EPA+DHA content, suggesting that the omega-3 index reflects the relative amounts of EPA+DHA in GI tissues. These data demonstrate that the GI tissues are highly responsive to dietary LCPUFA supplementation and that the omega- 3 index can serve as a valid biomarker for assessing dietary EPA+DHA incorporation into GI tissues.

\footnotetext{
(C) 2014 Elsevier Ltd. All rights reserved.

*Corresponding Author: Jenifer I Fenton, Department of Food Science and Human Nutrition, Michigan State University, 208B G.M. Trout Building, East Lansing, MI. 517-355-8474 ext 130. imigjeni@msu.edu.

Author Disclosure: WSH is the President of OmegaQuant Analytics, LLC and a Senior Research Scientist at Health Diagnostic Laboratory, Inc. Both of these laboratories offer omega-3 index testing, the former for researchers and the latter for clinicians. He is also a consultant to companies with interests in omega-3 fatty acids (Omthera, Amarin, Aker and Biomarine Antarctica).

AUTHORSHIP

E.G. and J.I.F. designed the research, interpreted results, and wrote the paper. J.W., S.H., W.H. performed experiments. E.G. and C.M. analyzed data and performed statistical analysis. All authors reviewed and provided input towards final paper. J.I.F. was responsible for the final content.

Publisher's Disclaimer: This is a PDF file of an unedited manuscript that has been accepted for publication. As a service to our customers we are providing this early version of the manuscript. The manuscript will undergo copyediting, typesetting, and review of the resulting proof before it is published in its final citable form. Please note that during the production process errors may be discovered which could affect the content, and all legal disclaimers that apply to the journal pertain.
} 


\section{INTRODUCTION}

Besides the purported cardiovascular benefits of omega-3 (n-3) long chain polyunsaturated fatty acids (LCPUFAs) found in fish, n-3 LCPUFA supplementation has been widely used in the treatment of inflammatory bowel diseases (IBD). Despite numerous clinical trials investigating the effect of n-3 LCPUFA supplementation on gastrointestinal (GI) diseases, no clear and consistent effect of fish oil on ulcerative colitis (UC) has been observed [1]. Pre-clinical diets high in fish and seafood may even increase the risk of developing UC and Crohn's Disease (CD) [2]. Furthermore, in animal models of colitis, treatment with fish oil has shown both beneficial [3,4] and adverse [5-8] health outcomes. Aside from disparate models of colitis induction, inconsistent results may be due to varying dose, type, and duration of fish oil exposure. Currently, there is no widely utilized biomarker of GI incorporation of n-3 LCPUFAs, further limiting comparability between studies.

Studies investigating the effect of n-3 LCPUFAs on cardiovascular health outcomes use the n-3 LCPUFA levels of red blood cells (RBCs) as a biomarker of dietary n-3 LCPUFA intake. The omega-3 index, defined as the sum of eicosapentaenoic acid (EPA) + docosahexaenoic acid (DHA) in RBCs expressed as a percentage of total fatty acids [9], is a standard for measuring n-3 LCPUFA status in vivo. RBCs are longer-lived than platelets and lipoproteins and thus their fatty acid composition is more stable and reflective of chronic n-3 status (much like hemoglobin A1c is a better marker of glycemic status than plasma glucose) [10]. The omega-3 index was originally suggested as an inverse marker of risk for death from coronary heart disease (CHD) and has many characteristics of a risk factor [11]. In a human fish oil supplementation study identifying surrogate tissues for the assessment of n-3 LCPUFA in cardiac tissue, RBCs, plasma, and cheek cells responded to supplementation of $1 \mathrm{~g}$ EPA+DHA for 6 months with increasing incorporation of n-3 LCPUFAs [12]. The authors observed that the levels of n-3 LCPUFAs in RBCs remain highly correlated to the levels of n-3 LCPUFAs found in cardiac tissue both before and after supplementation with fish oil, despite significant differences in the absolute n-3 LCPUFA levels between the tissues. The baseline EPA+DHA in both the RBCs $(4.7 \%)$ and cardiac tissue $(1.7 \%)$ of patients increased on average by $\sim 100 \%$ (RBC $9.0 \%$; cardiac tissue $2.7 \%$ ) demonstrating comparable responses to long-term supplementation. Thus, the n-3 LCPUFA levels in RBCs are not only reflective of dietary n-3 LCPUFA intake, but also serve as a surrogate biomarker of n-3 LCPUFA levels in cardiac tissue [11].

It is unclear, however, whether the omega-3 index adequately reflects the n-3 LCPUFA levels in tissues other than the heart. With inconsistent outcomes among studies assessing the effects of fish oil supplementation on gut health, a surrogate biomarker of n-3 LCPUFA incorporation for GI tissues may help identify discrepancies that stem from differences in study design (dose, duration, type of fish oil, etc). Therefore, the aim of our research was to investigate whether the fatty acid composition of RBCs (omega-3 index) reflects that of GI tissues. We supplemented the diets of colitis-prone mice with different concentrations of n-3 LCPUFAs (by percent energy in the diet, \%en). The doses of EPA+DHA in the murine diets were based upon recommenations by the American Heart Association (AHA) for consumption of fish or fish oil supplementation in humans [13]. These doses translated to 
$0.1 \%$ en, $0.675 \%$ en, and $1.8 \%$ en from dietary EPA+DHA. Therefore, for someone consuming a $2000 \mathrm{kcal}$ diet, whereby $30 \%$ of energy is derived from fat, these diets equate to $250 \mathrm{mg}, 1500 \mathrm{mg}$, and $4000 \mathrm{mg}$ EPA+DHA per day. We assessed the acyl chain composition of phospholipid fatty acids from RBCs, plasma, and several GI tissues (stomach, small intestine, cecum, and colon) to determine whether the RBC is a valid surrogate biomaker for plasma and GI FA composition across increasing intakes of n-3 LCPUFAs.

\section{MATERIALS AND METHODS}

\section{Dietary Treatment}

Experimental diets contained increasing amounts of EPA+DHA-enriched fish oil. The pharmaceutical fish oil, Lovaza, contains $465 \mathrm{mg}$ EPA and $375 \mathrm{mg}$ DHA per 1-g fish oil capsule; therefore, we created an EPA+DHA-enriched fish oil that was not predominantly either EPA or DHA. An EPA+DHA-enriched oil was made by combining appropriate volumes of EPA-enriched and DHA-enriched fish oils (Ocean Nutrition Canada, Dartmouth, Nova Scotia, Canada) until a 1:1 ratio of EPA:DHA was established. The oils were free of toxins, heavy metals, and oxidation products. The composition of the diets is detailed in Supplementary Table 1 and the fatty acid compositions in Supplementary Table 2. Using standard AIN-93G as the base diet, all diets contained $7 \%$ fat by weight, providing a total of $16.7 \% \mathrm{Kcal}$ of energy from fat. The control diet contained $7 \%$ weight-by-weight (wt/wt) soybean oil, while the experimental diets substituted a portion of the soybean oil with the EPA+DHA-enriched fish oil. The control diet modeled no intake of EPA or DHA. The following recommendations by the AHA for human intakes of fish and fish oil were converted to murine diets on a \%en basis. For example, individuals without documented heart disease or dyslipidemia, $250 \mathrm{mg}$ EPA+DHA approximates the n-3 LCPUFA content from the current AHA recommendation to consume at least 2 servings of fish a week. The medium n-3 LCPUFA treatment models the 1000-1500 mg EPA+DHA recommendation from the AHA for patients with documented heart disease, whereas the high n-3 LCPUFA intake models a 4000 mg EPA+DHA prescription (Lovaza) for patients with high triglycerides. Therefore, the human doses that were chosen for this study were $250 \mathrm{mg}, 1500$ $\mathrm{mg}$, and $4000 \mathrm{mg}$ EPA+DHA and were based on a standard $2000 \mathrm{kcal} \mathrm{human}$ diet with $30 \%$ energy (\%en) from fat. These doses translate to $0.1 \%$ en, $0.675 \%$ en, and $1.8 \%$ en from dietary EPA+DHA. To verify accuracy of the EPA:DHA ratio and increasing n-3 LCPUFA content of the diets, the fatty acid composition of the diet was measured by gas chromatography (Supplementary Table 2). Mice were fed the experimental diets ad libitum for 5 wk. Diets were kept frozen and changed daily.

\section{Experimental Design}

All animals were housed under specific pathogen-free (SPF) conditions in 60-square-inch plastic microisolator cages in the Research Containment Facility at Michigan State University, which is accredited by the Association for Assessment and Accreditation of Laboratory Animal Care. All animal procedures were previously approved by the Michigan State University All-University Committee on Animal Care and Use. A total of twenty-four male and female, 5-wk-old SMAD3-/- mice ( $\mathrm{n}=6 /$ treatment) were fed either control or one 
of the three EPA+DHA diets for $5 \mathrm{wk}$. One of the goals of our lab is to investigate the immunomodulatory effects of fish oil feeding in the SMAD3-/- mouse model of infectioninduced colitis. In the absence of infection, SMAD3-/- mice are otherwise healthy and remain free of colitis. To keep consistent with previous studies, we used this mouse model under the assumption that knockout of SMAD3, a transcription factor for TGF- $\beta$ signalling, does not affect fatty acid uptake and membrane remodeling. After dietary treatment, mice were asphyxiated with $\mathrm{CO}_{2}$ and exsanguinated through cardiac puncture using a heparincoated syringe. Tissues were promptly removed, rinsed in cold phosphate buffered saline and placed into sterile microcentrifuge tubes before freezing on dry ice. To obtain red blood cell (RBC) and plasma fractions, whole blood was centrifuged at $13,000 \times \mathrm{g}$ for $10 \mathrm{~min}$ at $4^{\circ} \mathrm{C}$ and collected by aspiration into separate tubes. All samples were then transferred under dim lighting into opaque containers for $-80^{\circ} \mathrm{C}$ storage until lipids were extracted.

\section{Extraction of Tissue and Erythrocyte Lipids}

Lipid extraction was performed using a modified version of the Rose and Oaklander method [14], which utilizes a mixture of 2-propanol:chloroform (11:7 v/v) to minimize heme interferences within erythrocytes or heme-contaminated tissues [15]. To ensure uniform sampling from each tissue, lipid extraction was preceded by pulverizing frozen tissue (100$200 \mathrm{mg}$ tissue/sample) using a mortar/pestle on dry ice. Under dim lighting, frozen powdered tissue, packed erythrocytes and plasma fractions were transferred into individual $16 \times 100 \mathrm{~mm}$ Teflon-lined screw-capped glass tubes and immediately resuspended in $2 \mathrm{~mL}$ of ice-cold HPLC-grade water (J.T. Baker, Phillipsburg, NJ). In the same vial, samples were homogenized for $30 \mathrm{~s}$ using a polytron homogenizer (Kinematica GmbH, Bohemia, NY), tightly capped, and incubated on ice for $15 \mathrm{~min}$. Tissues were then combined with $6 \mathrm{~mL}$ of 2-propanol (Sigma - Aldrich, St. Louis, MO) containing $100 \mu \mathrm{g} / \mathrm{mL}$ butyrated hydroxytoluene (BHT; Sigma - Aldrich), vortexed and incubated for $1 \mathrm{~h}$ on ice with occasional mixing. Following incubation, $3.8 \mathrm{~mL}$ of HPLC-grade chloroform (OmniSolv, Charlotte, NC) was added and samples where incubated for an additional hour on ice with occasional mixing. Phases were divided by centrifugation at $1,800 \times \mathrm{g}$ for $30 \mathrm{~min}\left(4^{\circ} \mathrm{C}\right)$ in a swinging bucket rotor. The lower phase was placed into a new $16 \times 100 \mathrm{~mm}$ glass tube, while the remaining upper aqueous phase and interfacial protein disc were re-extracted with $3.8 \mathrm{~mL}$ of chloroform for $1 \mathrm{~h}$ on ice with occasional vortexing. Following centrifugation, the lower phases were combined and dried under a gentle stream of nitrogen at $40^{\circ} \mathrm{C}$ prior to solid-phase extraction.

\section{Solid-Phase Extraction of Phospholipids}

Under minimal lighting, phospholipid isolation using solid-phase extraction was performed according to the modified procedures of Agren et al. [16]. Isolute-XL® SPE aminopropyl columns (500 mg; Biotage, Charlotte, NC) were contidioned twice with $5 \mathrm{~mL}$ acetone:water $(7: 1 \mathrm{v} / \mathrm{v})$ and activated twice with $4 \mathrm{~mL} n$-hexane (high purity solvent, OmniSolv, Charlotte, $\mathrm{NC}$ ). Dried lipids were dissolved in $2 \mathrm{~mL}$ of $n$-hexane:chloroform:acetic acid (100:5:5 v/v/v) containing $100 \mu \mathrm{g} / \mathrm{mL}$ BHT. Lipid samples were then added to individual columns monitored to prevent them from drying. To remove neutral lipids and non-esterified free fatty acids (NEFA), columns containing a sample were washed with $2 \mathrm{~mL}$ of $n$-hexanes, followed by $5 \mathrm{~mL}$ of $n$-hexane:chloroform:ethyl acetate (100:5:5 v/v/v; $100 \mu \mathrm{g} / \mathrm{mL}$ BHT), 
and $5 \mathrm{~mL}$ methanol:chloroform:acetic acid (100:2:2 v/v/v; $100 \mu \mathrm{g} / \mathrm{mL}$ BHT). Collection tubes were placed on ice under the columns and phospholipids were eluted twice with $4 \mathrm{~mL}$ of methanol:chloroform:water (100:5:4 v/v/v; $100 \mu \mathrm{g} / \mathrm{mL} \mathrm{BHT)}$ and $1 \mathrm{~mL}$ high purity methanol (Burdick \& Jackson, Morristown, NJ) to remove any residual phospholipids [17]. The collection tubes were brought to volume with chloroform, centrifuged at 2,000 $\times \mathrm{g}$ for $15 \mathrm{~min}\left(4^{\circ} \mathrm{C}\right)$ to remove any residual water before completely drying solvent fractions under a gentle stream of nitrogen at $40^{\circ} \mathrm{C}$.

\section{Preparation of Fatty Acid Methyl Esters}

Fatty acid methyl esters (FAMEs) were prepared from isolated phospholipids fractions by incubation with acidified methanol, according to the methods of Burdge et al. [18]. Dried phospholipids were mixed in $1 \mathrm{~mL}$ of toluene (J.T. Baker, Phillipsburg, NJ) by vortexing for $15 \mathrm{~s}$. A $3 \mathrm{~mL}$-aliquot of high purity methanol containing $2 \%$ sulfuric acid (J.T. Baker) was then added, gently mixed and incubated under minimal lighting at $55^{\circ} \mathrm{C}$ for $16 \mathrm{~h}$. The reaction mixture was cooled and neutralized with $3 \mathrm{~mL}$ of a solution containing $250 \mathrm{mM}$ potassium bicarbonate and $500 \mathrm{mM}$ potassium carbonate dissolved in HPLC grade water. Phospholipid FAMEs were isolated by the addition of $5 \mathrm{~mL}$ high purity $n$-hexane (containing $100 \mu \mathrm{g} / \mathrm{mL}$ BHT) and the separation of phases by centrifugation at 2,000 $\times \mathrm{g}$ for $15 \min \left(10^{\circ} \mathrm{C}\right)$. The upper $n$-hexane layer was removed, and dried under a gentle stream of nitrogen at room temperature. Isolated phospholipid FAMEs were resuspended in 1-8 $\mu \mathrm{L} / \mathrm{mg}$ tissue of $n$-hexane ( $100 \mu \mathrm{g} / \mathrm{mL}$ BHT), transferred to a GC autosampler vial, and stored under nitrogen at $-80^{\circ} \mathrm{C}$ until analysis.

\section{Fatty Acid Methyl Ester Analysis, Identification and Quantification}

As described previously [9], tissue and erythrocyte fatty acid compositions were analyzed using the HS-Omega-3 Index ${ }^{\circledR}$ methodology at OmegaQuant Analytics, LLC) (Sioux Falls, SD). Phospholipid fatty acid methyl esters were measured by gas chromatography using a GC2010 Gas Chromatograph (Shimadzu, Columbia, MD) equipped with a SP2560, 100-m column (Supelco, Bellefonte, PA) using hydrogen as carrier gas. Fatty acids were identified by comparison with a standard mixture of fatty acids characteristic of erythrocytes.

Phospholipid fatty acids were calculated as a percentage of total identified fatty acids after response factor correction. The omega-3 index is erythrocyte EPA plus DHA expressed as weight percentage of total fatty acids $[19,20]$. The coefficient of variation for the omega-3 index was $<5 \%$.

\section{Statistical analysis}

Significant differences in fatty acid levels between diets for a given tissue were determined using a one-way ANOVA followed by a Tukey post-hoc test. Significant differences in fatty acid levels between tissues for a given diet were determined using a one-way ANOVA followed by a Tukey post-hoc test. Within each treatment the data were normally distributed. Pearson correlation coefficients were calculated between RBC vs. plasma and RBC vs. GI tissues for specific fatty acids. Differences with $P$ values of $<0.05$ were considered statistically significant. All statistical analyses were conducted using the GraphPad Prism 4 software (GraphPad, San Diego, CA). 


\section{RESULTS}

\section{Diets and the Omega-3 Index}

Our first aim was to determine the range of the omega-3 index in mice fed diets reflective of current recommendations for human consumption of fish and/or fish oil supplementation. The omega-3 index increased in a dose-response fashion from $4.5 \%$ in mice fed the $0.0 \%$ en EPA+DHA diet to $\sim 11 \%$ in mice fed the $1.8 \%$ en EPA+DHA diet (Figure 1A). As the \%en from EPA+DHA increased in the diet, the omega-3 index, plasma EPA+DHA, and GI tissue EPA+DHA increased $(\mathrm{p}<0.05)$ (Table 1). Within the control diet, the omega-3 index and the EPA+DHA content of the small intestine, cecum and colon did not significantly differ. However, the baseline plasma EPA+DHA (6.13\%) was significantly higher than the omega-3 index (5.22\%), whereas the stomach EPA+DHA (2.34\%) was significantly lower. Within the 1.8\% en EPA+DHA diet, the EPA+DHA content of the plasma, small intestine, and colon were significantly higher $(13.14-14.07 \%)$, whereas the stomach remained significantly lower (7.34\%) compared to the cecum (11.54\%) and omega-3 index (11.25\%).

To determine whether the changes in the omega-3 index (Figure 1A) reflect changes in the plasma and gastrointestinal tract tissues after 5 weeks of feeding, we calculated the Pearson correlation coefficient to test for linear correlations between the omega-3 index and the EPA +DHA content of the other tissues (Figure 1B-F). Indeed, strong $(r>0.91)$ and significant $(\mathrm{p}<0.001)$ correlations were observed between the omega-3 index and the EPA+DHA content for all other tissues.

\section{LCPUFA Correlations between RBC and plasma or GI tissues}

The primary n-3 and n-6 LCPUFAs to change (i.e. the fatty acids that changed the most drastically) were EPA, DHA, and arachidonic acid (AA), whereas minor, albeit significant, changes were observed in other LCPUFAs. The data for all fatty acids from the phospholipids of RBCs, plasma, stomach, small intestine, cecum, and colon across all dietary regimens are provided in (Supplementary Tables 3-8). We were next interested in whether any individual n-3 or n-6 LCPUFA were as strongly correlated between RBCs and plasma and RBCs and GI tissues. RBC EPA was strongly correlated with both plasma EPA and GI tissue EPA, as was the omega-3 index was to plasma/GI EPA+DHA content with correlation coefficients all > 0.91 (Table 2). However, RBC DHA was strongly correlated with all other tissue DHA, but was less well correlated to plasma DHA $(r=0.752)$ and stomach $(r=0.846)$. RBC AA was also strongly correlated with all other tissue AA, but the correlation was less strong, with all correlations coefficients between 0.81 and 0.89 .

\section{EPA, DHA, and AA fold increases}

We then calculated the fold-change of EPA, DHA, and AA for each mouse on the experimental diets containing EPA+DHA-enriched fish oil over the respective amount of LCPUFA in the tissues of mice in the control group. Using the fold-change over the control group as an approximation of the expected relative rate of enrichment after five weeks of feeding a control animal at the respective dose, we evaluated n-3 LCPUFA uptake across tissues. 
We observed that the RBCs, plasma, stomach, and small intestine took up significantly more EPA than the colon and cecum, regardless of the diet; e.g. the 1.8\% EPA+DHA diet, the RBCs, plasma, stomach, and small intestine contained approximately $9-11$ times more EPA than their control counterparts, compared to approximately $4-6$ times more within the colon and cecum. When correlating the EPA fold-change of RBCs to the fold change of EPA in other other tissues, we observed highly significant correlations between tissues over the ranges of doses provided (plasma data not shown, slope $\sim 1.0, r>0.70$, and $p<0.01$ ) (Figure 2A-D). This correlation determines whether the saturation of RBC EPA increases in a similar fashion to that of plasma and GI EPA saturation. Differences in the saturation of tissues may be due to the baseline absolute levels; at baseline, similar differences in the absolute levels of EPA between tissues from mice on the 0.0\%en EPA+DHA diet $(0.2-$ $0.6 \%$ ) remain apparent after increases in EPA in mice fed the 1.8\%en EPA+DHA diet (1.64.3\%) (Figure 2E-F).

The fold-change increases of DHA across all tissues were considerably smaller compared to the fold-changes of EPA, resulting in approximately 1.6 - 2.7 times as much DHA in tissues from mice on the $1.8 \%$ en EPA+DHA diets compared to those on the control diet. Still, there were significant differences in the fold-change of DHA between tissues within any given diet. For the mice on the 1.8\% en EPA+DHA diet, the fold-change of DHA in RBCs (1.6) and plasma (1.7) was considerably lower than the stomach (2.6) and small intestine (2.3). Comparably the fold-changes of DHA in the cecum (2.2) and colon (2.0) were in-between the aforementioned tissues, often with insignificant comparisons. When correlating the DHA fold-change of RBCs to that of other tissues, we observed highly significant correlations between tissues over the ranges of doses provided (plasma data not shown, slope $\sim 1.0, r>$ 0.70 , and $\mathrm{p}<0.01$ ) (Figure 3A-D). This correlation determines whether the saturation of RBC DHA increases in a similar fashion to that of plasma/GI DHA saturation. Differences in the saturation of tissues may be due to the baseline absolute levels; at baseline, similar differences in the absolute levels of DHA between tissues from mice on the 0.0\%en EPA +DHA diet $(2.2-5.9)$ remain apparent after increases in DHA in mice fed the 1.8\%en EPA +DHA diet $(5.8-10.5 \%)$ (Figure 3E-F).

Consistent with the literature and despite the increasing amount of AA in the EPA+DHAenriched fish oil diets, AA was displaced from phospholipid fatty acids as the dose of EPA +DHA increased. Notably, the $0.1 \%$ en and $0.675 \%$ en EPA+DHA diets did not induce the same displacement of AA when comparing between tissues. However, for all tissues from mice on the $1.8 \%$ en EPA+DHA diet, AA was $0.50-0.63$ times less than the control diet with no significant differences observed between tissues. When correlating the AA foldchange of RBCs to that of other tissues, we observed highly, significant correlations between tissues over the ranges of doses provided (plasma data not shown, slope $\sim 1.0, r>$ 0.70 , and $\mathrm{p}<0.01$ ) (Figure 4A-D). This correlation determines whether the displacement of RBC AA increases in a similar fashion to that of plasma/GI AA displacement. Differences in the displacement of tissue AA may be due to the baseline absolute levels; at baseline, similar differences in the absolute levels of AA between tissues from mice on the $0.0 \%$ en EPA+DHA diet $(6.9-14.1 \%)$ remain apparent after increases in AA in mice fed the $1.8 \%$ en EPA+DHA diet $(3.9-8.5 \%)$ (Figure 4E-F). 


\section{DISCUSSION}

We found that, regardless of the organ, GI tissue EPA+DHA and EPA levels were very highly correlated with the RBC levels, whereas DHA and AA levels were also signifiantly correlated but somewhat less strongly across all tissues. Accordingly, the omega-3 index may be used as a valid surrogate for the relative enrichment of GI tissue EPA+DHA content. We observed that small amount of dietary EPA+DHA (at 0.1\%en EPA+DHA) resulted in increased n-3 LCPUFA levels in blood and GI tissues. These data demonstrate the capacity of RBC fatty acids to act as a surrogate biomarker for assessing n-3 LCPUFA incorporation in GI tissues. While the omega-3 index adequately predicts the relative enrichment of EPA +DHA in GI tissues, we observed that the omega-3 index does not reflect the absolute EPA +DHA in GI tissues. In addition, we observed significant disparities in the levels of incorporation between tissues for the primary n-3 LCPUFAs, EPA and DHA, suggesting that rate of enrichment and saturation may be tissue dependent. These findings indicate that the RBC does not mirror the absolute levels of $n-3$ and n-6 LCPUFAs in GI tissue, but the RBC does reflect the relative enrichment of n-3 and n-6 LCPUFAs in GI tissues.

The clinical utility of the omega- 3 index was demonstrated in previous studies showing that the omega-3 index may be a predictor of actual risk for CHD [11]. This may be due in part due to the fact that the omega-3 index is reflective of the cardiac tissue EPA+DHA [11]. Meta-analyses of clinical trials on fish oil supplementation as a potential therapeutic for various GI diseases, including IBD, have been conducted, but are often inconclusive [1, 21]. Given the often disparate exposure of n-3 LCPUFAs (e.g. type of fish oil, duration, etc.), it is important to know the extent to which n-3 LCPUFAs are actually incorporated into the GI tissues of interest. Our data suggest that the omega-3 index will, at least in mice, provide a valid estimate of GI tissue EPA and DHA levels.

Previous work by Arnold et al. examined the fatty acid composition of various tissues of Zucker rats fed a diet comparable in fat composition to the AIN-93G or a fish oil supplemented AIN-93G (where 4.4\%en was derived from EPA+DHA) for three weeks [22]. In the animals fed the control diets containing $0.0 \%$ en from EPA+DHA in both studies, the omega-3 index for the Zucker rats' were $1.6 \%$ in RBCs and $1.8 \%$ in plasma, compared to our study where the omega-3 index was $5.2 \%$ in RBCs and $6.1 \%$ in plasma. Consistent with having comparatively lower levels of n-3 LCPUFAs in tissues of animals fed their control diets, they observed higher AA in the RBCs (26.6\%) and plasma (10.5\%), than we had observed in our control diet (RBCs, 11.3\%; plasma, 6.3\%). While we had observed decreases in n-6 LCPUFAs and increases in n-3 LCPUFAs with increasing dietary EPA +DHA, the absolute levels of n-3/6 LCPUFAs of either RBCs or plasma remained considerably different between the studies. The high \%en EPA+DHA groups resulted dramatic reduction of AA in the Arnold et al. study (RBC, 17.1\%; plasma, 3.1\%) and the data presented here (RBC, 5.8\%; plasma, 3.9\%). Unsurprisingly, the high \%en EPA+DHA groups also had significant increases in the omega-3 index in the Arnold et al. study (RBC, 12.2\%; plasma, 7.6\%) and the data presented here (RBC, 11.2\%; plasma, 13.2\%). It is worth noting that the fatty acid levels of the Zucker rats in the Arnold et al. study increased in EPA content to a greater degree, despite having similar levels between the studies in the $0.0 \%$ en EPA+DHA diets. This difference, as well as others, may be due to the fact that Arnold et al. 
had extracted total tissue lipid, whereas we had isolated the phospholipid fraction of all tissues. Despite the differences in absolute levels of n-3/6 LCPUFAs between studies, the similar type of supplementation ( 1:1 EPA:DHA) in the Arnold et al. study provides complimentary evidence to support that RBCs and plasma undergo similar changes in tissue-extracted lipid/phospholipid after inclusion of EPA+DHA in the diet in a relatively short period of time.

We had identified at least one other study that reported the fatty acid composition of membrane phospholipids from the small intestine of mice fed standard AIN-93G-based diet [23]. The diets were supplemented with oleic acid, AA, EPA, or AA+EPA for 8 wks. Consistent with the reported values in their control group, the absolute levels of most major fatty acids (16:0, 18:0, 18:1, 18:2n-6, 20:5n-3, 22:6n-3) reported were similar to the levels obtained in our control group. AA was the only fatty acid that was considerably higher when comparing their findings (16.6\%) to ours (12.8\%). Because they had used only EPA for the n-3 LCPUFA treatment, direct comparison to our EPA+DHA treatments is not possible. Together these data demonstrate consistency of phospholipid fatty acid analysis of tissues across mouse models.

In the present study, we investigated the effect of increasing EPA+DHA content of soybean oil-based AIN-93G; however, others have investigated the lipid composition of various tissues from mice fed diets with non-standard lipid mixtures [7, 24, 25]. At least two studies that have reported the fatty acid composition of colonic mucosa where the lipid in the diet was primarily low in alpha-linolenic (ALA; 18:3n-3), such as canola or corn oil, have observed low levels of DHA (1.3\% [24], 1.6\% [7]. This contrasts our reported values for mice fed the $0.0 \%$ en diet, whereby the DHA content in the membrane of colonic tissue was found to be $\sim 5.0 \%$ of all phospholipids. The higher level of DHA observed in our study may be due to the use of soybean oil, in contrast to the use of corn oil in their diets. In mice eating diets devoid of fish oil, the level of EPA present in the colon was reported to be low by both us and Turk et al., regardless of base oil composition. Their observations corroborate ours in separate mouse models increasing generalizability of colonic fatty acid composition across murine models.

The use of a non-human model to measure LCPUFA incorporation into tissue is a potential limitation to this study. However, testing the effects of escalating levels of dietary omega-3 on solid organ fatty acid composition in humans is virtually impossible. Therefore, in the present study we assessed the phospholipid fatty acid composition of blood and GI tissues from mice to determine how well blood correlates with GI tissues. There are a number of studies that confirm a strong relationship between intake and the omega-3 index in humans suggesting a high degree of comparability, allowing for reasonable extrapolation of our findings. Specifically, the RBC [12, 26], and plasma [12] LCPUFA levels of healthy humans and those found in mice fed AIN-93G display strong similarities, with a common theme of slightly lower DHA in human RBC and plasma (3.1 - 4.2\% and 2.5\%, respectively) compared to mice (4.9\% and 5.8\%, respectively). The $1.8 \%$ en EPA+DHA fed mice had approximately $8 \%$ DHA in RBCs, which parallels similarly to recent human supplementation studies that found DHA reaching levels of 7.0 - 7.5\% in RBCs [27, 28]. Furthermore, increasing research in humans demonstrate that the RBC may serve as a 
surrogate biomarker for the level of LCPUFAs in certain tissues, such as cardiac [12, 27, 29] and retina or optic nerves [26], but not others, such as the brain [30]. Indeed, in this mouse model we also observe a strong correlation between RBC and cardiac tissue n-3 LCPUFAs (data not shown).

A number of human studies have investigated whether the fatty acid composition of GI tissues in individuals with IBD differ compared to healthy controls, providing insight into the levels of n-3 and n-6 LCPUFAs found in human colonic mucosa [31-33]. Comparison between mouse and human studies reveal that the levels of n-3/6 LCPUFAs in RBC and plasma are generally in agreement between species, with slightly decreased levels of DHA in humans $(2.4-3.5 \%)$ compared to mice $(4.9 \%)$ and slightly decreased AA in humans $(9.0$ - 9.8\%) compared to mice (13.5\%). In humans consuming either a typical diet (baseline, pre-intervention) or a healthy/mediterranean diet, the colonic n-3 fatty acid \% (unspecified n-3 FAs) ranged from $4.4-5.1 \%$ [34] similar to percentages observed in our mouse study. In another human study examining the effect of EPA supplementation in subjects with a history of adenomas, colonic EPA+DHA ranged from $2.7-3.4 \%$ of total fatty acids at baseline and supplementation increased it to $5.1 \%$. This increase correlated with significantly reduced proliferation and increased mucosal apoptosis [32]. Finally, in a study comparing mucosal n-3 and n-6 fatty acid in the colorectal adenoma-carcinoma sequence, the EPA+DHA ranged from $2.9-3.5 \%$ of total fatty acids [35]. Considering that the phospholipid fraction of cell membranes contain the majority of fatty acids in most tissues (i.e. non-adipose, non-steroid synthesizing tissues), these data highlight the similarity in fatty acid composition of various GI tissues between mice and humans. Taken together, the similarities observed between mouse and human fatty acid profiles provide evidence to potentially expand utility of RBC in predicting tissue-level incorporation of n-3/6 LCPUFAs. We believe that it is not unreasonable to extrapolate our general findings from the mouse model to humans recognizing that the precise dose-response curves may differ in these two mammalian species but the overall features are likely to be similar.

With additional studies addressing the role of n-3 LCPUFAs on tissue-specific health outcomes in humans (e.g. CHD, IBD), there is a need to define an easily accessible and valid biomarker of $n-3$ LCPUFA status in the tissue(s) of interest. Based on our findings, the omega-3 index appears to serve that function, at least for GI tissues. The extent to which the omega-3 index can serve as a reflection of other tissues (e.g., lung, bone, skeletal or smooth muscle, liver, kidney, brain, etc.) remains to be determined.

\section{Supplementary Material}

Refer to Web version on PubMed Central for supplementary material.

\section{Acknowledgments}

Financial support for this research was provided by: NIH R03CA162427 (to JIF).

The authors would like to acknowledge Jason Polreis and Laura Sanborn for performing part of the fatty acid analysis. 


\section{Abbreviations Used}

$\%$ en

AA

CD

CHD

DFO

DHA

EFO

EPA

FAME

GI

IBD

n-3 LCPUFA

NEFA

RBC

SMAD3

SPF

TGF- $\beta$

UC
Percent of energy

Arachidonic Acid

Crohn's disease

Coronary heart disease

DHA-enriched fish oil

Docosahexaenoic acid

EPA-enriched fish oil

Eicosapentaenoic acid

Fatty acid methyl ester

Gastrointestinal

Inflammatory bowel disease

n-3 long chain polyunsaturated fatty acid

Non-esterified fatty acid

Red blood cell

mothers against decapentaplegic homolog-3

specific pathogen-free

transforming growth factor-beta

Ulcerative colitis

\section{References}

1. Turner D, Steinhart AH, Griffiths AM. Omega 3 fatty acids (fish oil) for maintenance of remission in ulcerative colitis. Cochrane Database Syst Rev. 2007:CD006443. [PubMed: 17636844]

2. Hou JK, Abraham B, El-Serag H. Dietary intake and risk of developing inflammatory bowel disease: a systematic review of the literature. The American journal of gastroenterology. 2011; 106:563-573. [PubMed: 21468064]

3. Gil A. Polyunsaturated fatty acids and inflammatory diseases. Biomed Pharmacother. 2002; 56:388396. [PubMed: 12442911]

4. Chapkin RS, Davidson LA, Ly L, Weeks BR, Lupton JR, McMurray DN. Immunomodulatory effects of (n-3) fatty acids: putative link to inflammation and colon cancer. The Journal of nutrition. 2007; 137:200S-204S. [PubMed: 17182826]

5. Matsunaga H, Hokari R, Kurihara C, Okada Y, Takebayashi K, Okudaira K, Watanabe C, Komoto S, Nakamura M, Tsuzuki Y, Kawaguchi A, Nagao S, Itoh K, Miura S. Omega-3 fatty acids exacerbate DSS-induced colitis through decreased adiponectin in colonic subepithelial myofibroblasts. Inflammatory bowel diseases. 2008; 14:1348-1357. [PubMed: 18484673]

6. Ghosh S, DeCoffe D, Brown K, Rajendiran E, Estaki M, Dai C, Yip A, Gibson DL. Fish oil attenuates omega- 6 polyunsaturated fatty acid-induced dysbiosis and infectious colitis but impairs LPS dephosphorylation activity causing sepsis. PloS one. 2013; 8:e55468. [PubMed: 23405155]

7. Turk HF, Monk JM, Fan YY, Callaway ES, Weeks B, Chapkin RS. Inhibitory effects of omega-3 fatty acids on injury-induced epidermal growth factor receptor transactivation contribute to delayed 
wound healing. American journal of physiology Cell physiology. 2013; 304:C905-917. [PubMed: 23426968]

8. Woodworth HL, McCaskey SJ, Duriancik DM, Clinthorne JF, Langohr IM, Gardner EM, Fenton JI. Dietary fish oil alters T lymphocyte cell populations and exacerbates disease in a mouse model of inflammatory colitis. Cancer research. 2010; 70:7960-7969. [PubMed: 20798218]

9. Harris WS, Von Schacky C. The Omega-3 Index: a new risk factor for death from coronary heart disease? Preventive medicine. 2004; 39:212-220. [PubMed: 15208005]

10. Harris WS, Varvel SA, Pottala JV, Warnick GR, McConnell JP. Comparative effects of an acute dose of fish oil on omega-3 fatty acid levels in red blood cells versus plasma: Implications for clinical utility. Journal of Clinical Lipidology. 2013

11. Harris WS. The omega-3 index: clinical utility for therapeutic intervention. Curr Cardiol Rep. 2010; 12:503-508. [PubMed: 20809235]

12. Harris WS, Sands SA, Windsor SL, Ali HA, Stevens TL, Magalski A, Porter CB, Borkon AM. Omega-3 fatty acids in cardiac biopsies from heart transplantation patients - Correlation with erythrocytes and response to supplementation. Circulation. 2004; 110:1645-1649. [PubMed: 15353491]

13. Kris-Etherton PM, Harris WS, Appel LJ. C. Nutrition. Fish consumption, fish oil, omega-3 fatty acids, and cardiovascular disease. Arteriosclerosis, thrombosis, and vascular biology. 2003; 23:e20-30.

14. Rose HG, Oklander M. Improved Procedure for Extraction of Lipids from Human Erythrocytes. Journal of lipid research. 1965; 6:428. [PubMed: 14336214]

15. Lydic TA, Renis R, Busik JV, Reid GE. Analysis of Retina and Erythrocyte Glycerophospholipid Alterations in a Rat Model of Type 1 Diabetes. Jala-J Lab Autom. 2009; 14:383-399.

16. Agren JJ, Julkunen A, Penttila I. Rapid Separation of Serum-Lipids for Fatty-Acid Analysis by a Single Aminopropyl Column. Journal of lipid research. 1992; 33:1871-1876. [PubMed: 1479296]

17. Bondia-Pons I, Morera-Pons S, Castellote AI, Lopez-Sabater MC. Determination of phospholipid fatty acids in biological samples by solid-phase extraction and fast gas chromatography. $\mathrm{J}$ Chromatogr A. 2006; 1116:204-208. [PubMed: 16584741]

18. Burdge GC, Wright P, Jones AE, Wootton SA. A method for separation of phosphatidylcholine, triacylglycerol, non-esterified fatty acids and cholesterol esters from plasma by solid-phase extraction. Brit J Nutr. 2000; 84:781-787. [PubMed: 11177194]

19. Aarsetoey H, Aarsetoey R, Lindner T, Staines H, Harris WS, Nilsen DWT. Low Levels of the Omega-3 Index are Associated with Sudden Cardiac Arrest and Remain Stable in Survivors in the Subacute Phase. Lipids. 2011; 46:151-161. [PubMed: 21234696]

20. von Schacky C. The Omega-3 Index as a risk factor for cardiovascular diseases. Prostag Oth Lipid M. 2011; 96:94-98.

21. Turner D, Shah PS, Steinhart AH, Zlotkin S, Griffiths AM. Maintenance of remission in inflammatory bowel disease using omega-3 fatty acids (fish oil): a systematic review and metaanalyses. Inflammatory bowel diseases. 2011; 17:336-345. [PubMed: 20564531]

22. Arnold C, Markovic M, Blossey K, Wallukat G, Fischer R, Dechend R, Konkel A, von Schacky C, Luft FC, Muller DN, Rothe M, Schunck WH. Arachidonic acid-metabolizing cytochrome P450 enzymes are targets of \{omega $\}-3$ fatty acids. The Journal of biological chemistry. 2010; 285:32720-32733. [PubMed: 20732876]

23. Petrik MB, McEntee MF, Chiu CH, Whelan J. Antagonism of arachidonic acid is linked to the antitumorigenic effect of dietary eicosapentaenoic acid in Apc(Min/+) mice. The Journal of nutrition. 2000; 130:1153-1158. [PubMed: 10801912]

24. Oikarinen SI, Pajari AM, Salminen I, Heinonen SM, Adlercreutz H, Mutanen M. Effects of a flaxseed mixture and plant oils rich in alpha-linolenic acid on the adenoma formation in multiple intestinal neoplasia (Min) mice. The British journal of nutrition. 2005; 94:510-518. [PubMed: 16197574]

25. Alvheim AR, Malde MK, Osei-Hyiaman D, Lin YH, Pawlosky RJ, Madsen L, Kristiansen K, Froyland L, Hibbeln JR. Dietary linoleic acid elevates endogenous 2-AG and anandamide and induces obesity. Obesity (Silver Spring). 2012; 20:1984-1994. [PubMed: 22334255] 
26. Acar N, Berdeaux O, Gregoire S, Cabaret S, Martine L, Gain P, Thuret G, Creuzot-Garcher CP, Bron AM, Bretillon L. Lipid composition of the human eye: are red blood cells a good mirror of retinal and optic nerve fatty acids? PloS one. 2012; 7:e35102. [PubMed: 22496896]

27. Metcalf RG, James MJ, Gibson RA, Edwards JR, Stubberfield J, Stuklis R, Roberts-Thomson K, Young GD, Cleland LG. Effects of fish-oil supplementation on myocardial fatty acids in humans. The American journal of clinical nutrition. 2007; 85:1222-1228. [PubMed: 17490956]

28. Flock MR, Skulas-Ray AC, Harris WS, Etherton TD, Fleming JA, Kris-Etherton PM. Determinants of erythrocyte omega-3 fatty acid content in response to fish oil supplementation: a dose-response randomized controlled trial. Journal of the American Heart Association. 2013; 2:e000513. [PubMed: 24252845]

29. Metcalf RG, Cleland LG, Gibson RA, Roberts-Thomson KC, Edwards JR, Sanders P, Stuklis R, James MJ, Young GD. Relation between blood and atrial fatty acids in patients undergoing cardiac bypass surgery. The American journal of clinical nutrition. 2010; 91:528-534. [PubMed: 20089730]

30. Carver JD, Benford VJ, Han B, Cantor AB. The relationship between age and the fatty acid composition of cerebral cortex and erythrocytes in human subjects. Brain research bulletin. 2001; 56:79-85. [PubMed: 11704343]

31. Rix TA, Joensen AM, Riahi S, Lundbye-Christensen S, Overvad K, Schmidt EB. Marine n-3 fatty acids in adipose tissue and development of atrial fibrillation: a Danish cohort study. Heart. 2013; 99:1519-1524. [PubMed: 23945170]

32. Courtney ED, Matthews S, Finlayson C, Di Pierro D, Belluzzi A, Roda E, Kang JY, Leicester RJ. Eicosapentaenoic acid (EPA) reduces crypt cell proliferation and increases apoptosis in normal colonic mucosa in subjects with a history of colorectal adenomas. International journal of colorectal disease. 2007; 22:765-776. [PubMed: 17216221]

33. Esteve M, Navarro E, Klaassen J, Abad-Lacruz A, Gonzalez-Huix F, Cabre E, Ramos E, Condom E, Fernandez-Banares F, Pastor C, Humbert P, Marti-Rague J, Gassull MA. Plasma and mucosal fatty acid pattern in colectomized ulcerative colitis patients. Digestive diseases and sciences. 1998; 43:1071-1078. [PubMed: 9590424]

34. Sen A, Ren J, Ruffin MT, Turgeon DK, Brenner DE, Sidahmed E, Rapai ME, Cornellier ML, Djuric Z. Relationships between Serum and Colon Concentrations of Carotenoids and Fatty Acids in Randomized Dietary Intervention Trial. Cancer Prev Res (Phila). 2013; 6:558-565. [PubMed: 23592741]

35. Fernandez-Banares F, Esteve M, Navarro E, Cabre E, Boix J, Abad-Lacruz A, Klaassen J, Planas R, Humbert P, Pastor C, Gassull MA. Changes of the mucosal n3 and n6 fatty acid status occur early in the colorectal adenoma-carcinoma sequence. Gut. 1996; 38:254-259. [PubMed: 8801207] 

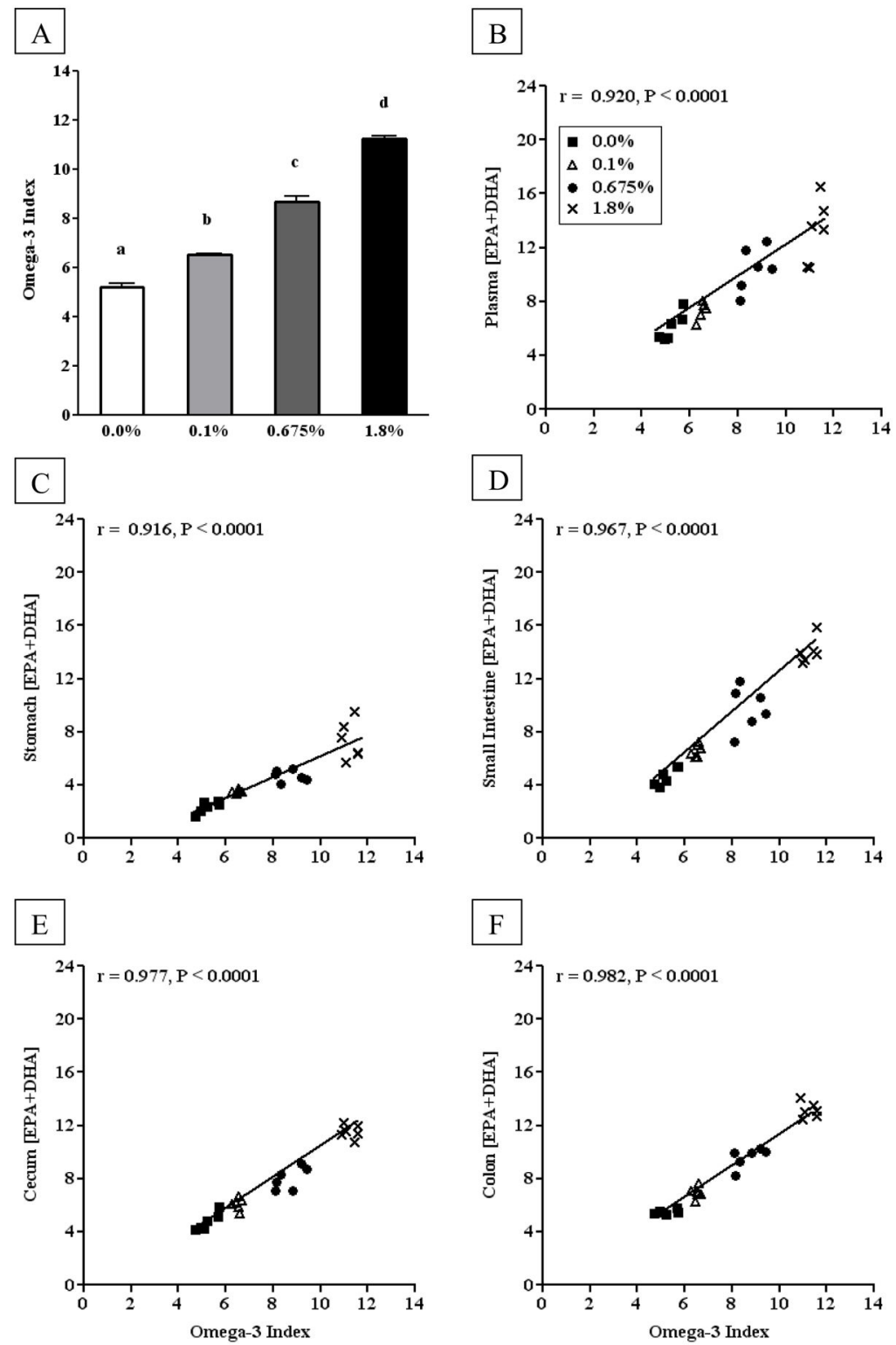

Figure 1. Correlations between the omega-3 index of red blood cell and the EPA+DHA of plasma or gastrointestinal tract tissues from mice fed diets with increasing EPA+DHA

(A) The omega-3 index from mice fed AIN-93G diet with either 0.0\%en EPA+DHA, $0.1 \%$ en EPA+DHA, $0.675 \%$ en EPA+DHA, or $1.8 \%$ en EPA+DHA ( $n=6 /$ group). A oneway ANOVA and a Tukey's post-hoc were used to assess differences in the tissue-specific EPA+DHA compared to each other. Differing letters denote statistical significance at $P<$ 0.05. (B-E) The omega-3 index in RBC phospholipids correlated to the EPA+DHA of plasma (B), stomach (C), small intestine (D), cecum (E), and colon (F) phospholipids. A Pearson correlation was used to test for linear correlation between the aforementioned tissues $(n=24)$. 

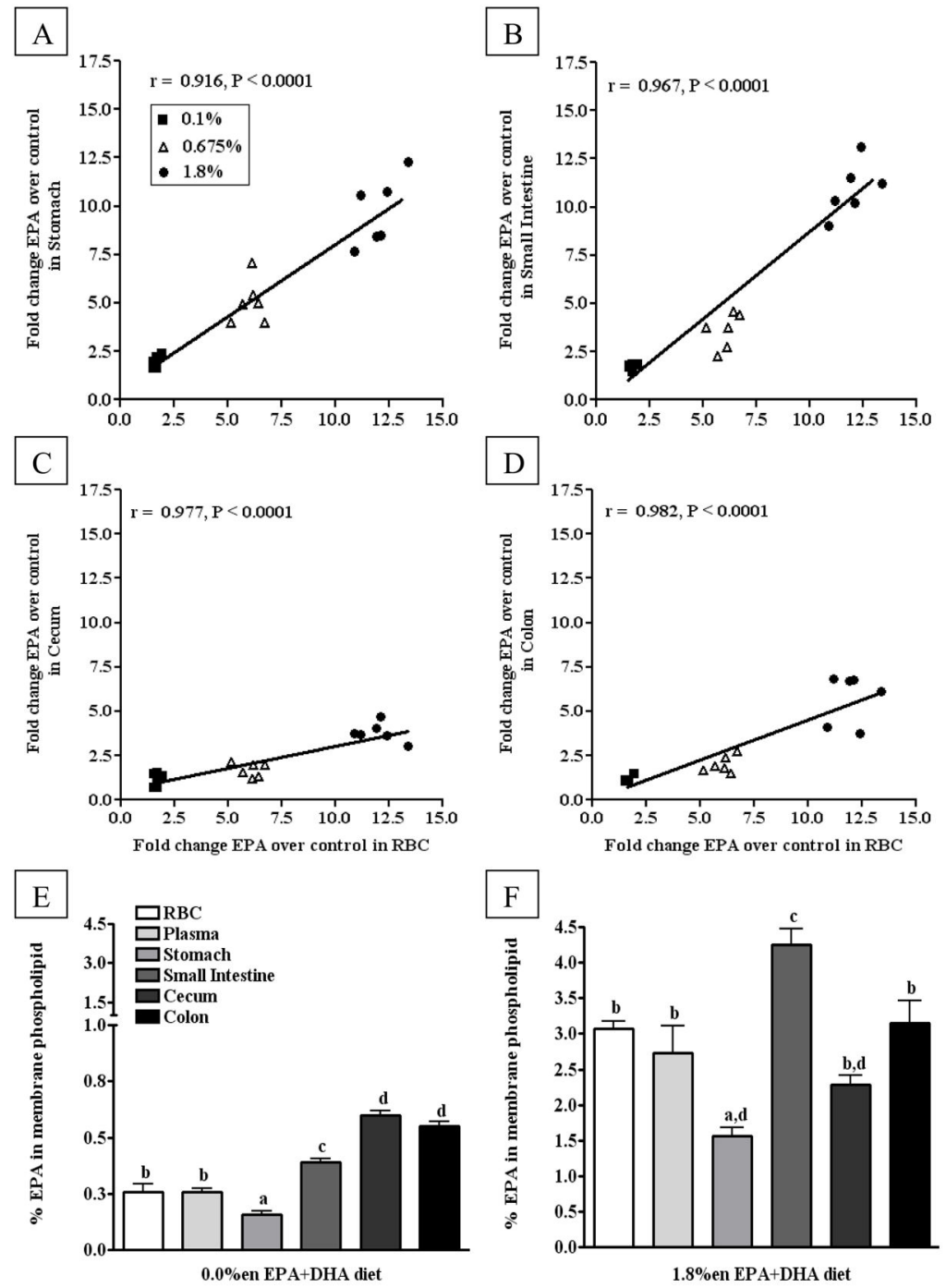

Figure 2. Comparison of EPA content in phospholipid fatty acids from blood and gastrointestinal tissues mice fed diets with increasing EPA+DHA

(A-D) Fold Change in EPA content over the control group in phospholipid fatty acids from gastrointestinal tissues correlated to EPA content in phospholipid fatty acids from red blood cells: (A) Stomach, (B) Small Intestine (C) Cecum, (D) Colon. Data from the tissues of mice fed experimental diets containing EPA+DHA-enriched fish oil were normalized to the $0.0 \%$ en EPA+DHA diet group to assess fold changes in EPA content of phospholipids. A Pearson correlation was used to test linear correlation between gastrointestinal tissue EPA fold change and RBC EPA fold change $(n=18)$. (E-F) The EPA content from phospholipid fatty acids across blood and gastrointestinal tissues from mice fed either the control (E) or 1.8\% en EPA+DHA diet (F) ( $\mathrm{n}=6$ /group). A one-way ANOVA and a Tukey's post-hoc were used to assess differences in EPA content comparing all tissues to each other. Differing letters denote statistical significance at $P<0.05$. 

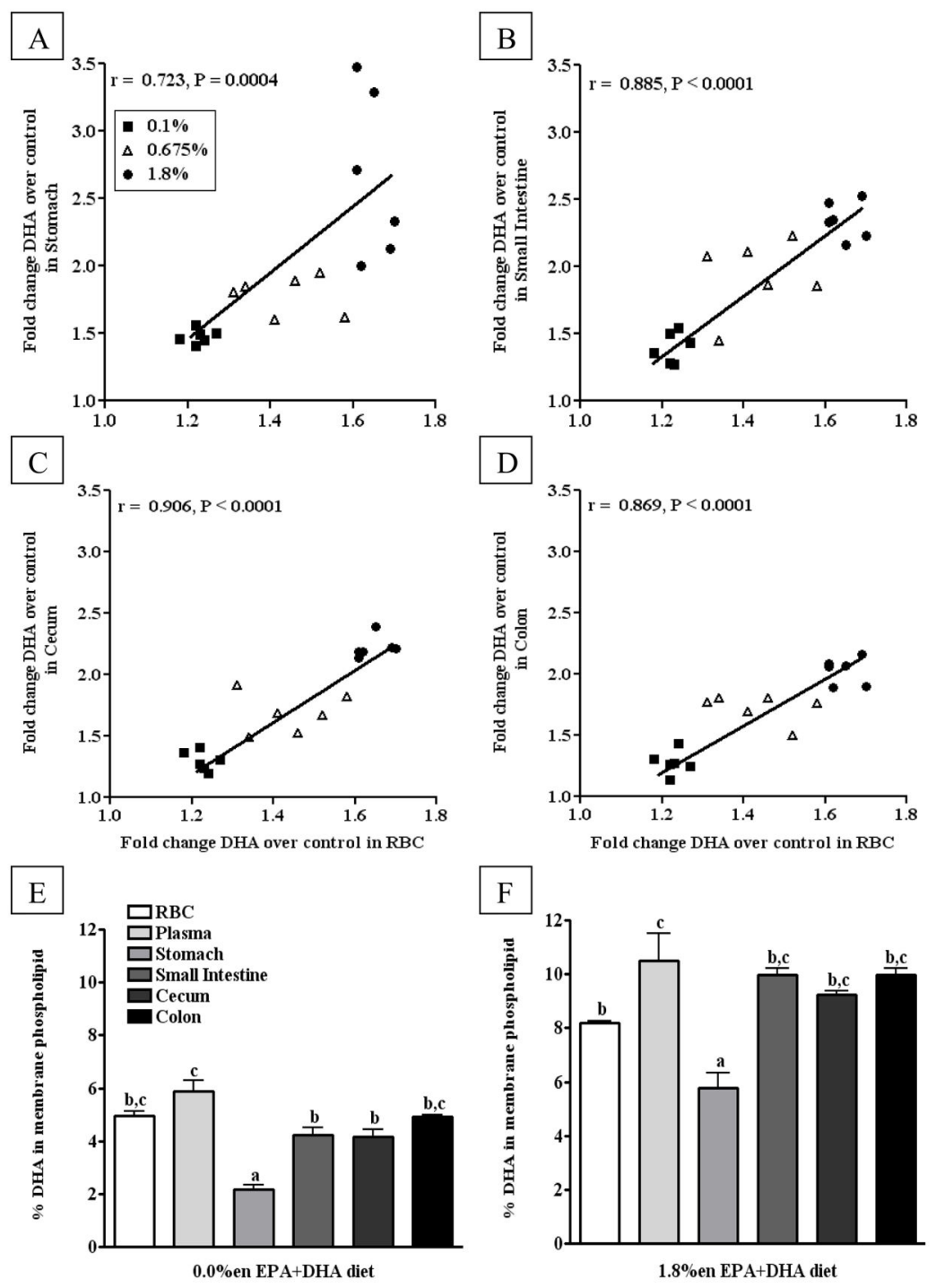

Figure 3. Comparison of DHA content in phospholipid fatty acids from blood and gastrointestinal tissues from mice fed diets with increasing EPA+DHA

(A-D) Fold Change in DHA content over the control group in phospholipid fatty acids from gastrointestinal tissues correlated to DHA content in phospholipid fatty acids from red blood cells: (A) Stomach, (B) Small Intestine (C) Cecum, (D) Colon. Data from the tissues of mice fed experimental diets containing EPA+DHA-enriched fish oil were normalized to the control group to assess fold changes in DHA content of phospholipids. A Pearson correlation was used to test linear correlation between gastrointestinal tissue DHA fold change and RBC DHA fold change $(\mathrm{n}=18)$. (E-F) The DHA content from phospholipid fatty acids across blood and gastrointestinal tissues from mice fed either the control (E) or $1.8 \%$ en EPA+DHA diet (F) ( $\mathrm{n}=6$ /group). A one-way ANOVA and a Tukey's post-hoc were used to assess differences in DHA content comparing all tissues to each other. Differing letters denote statistical significance at $P<0.05$. 

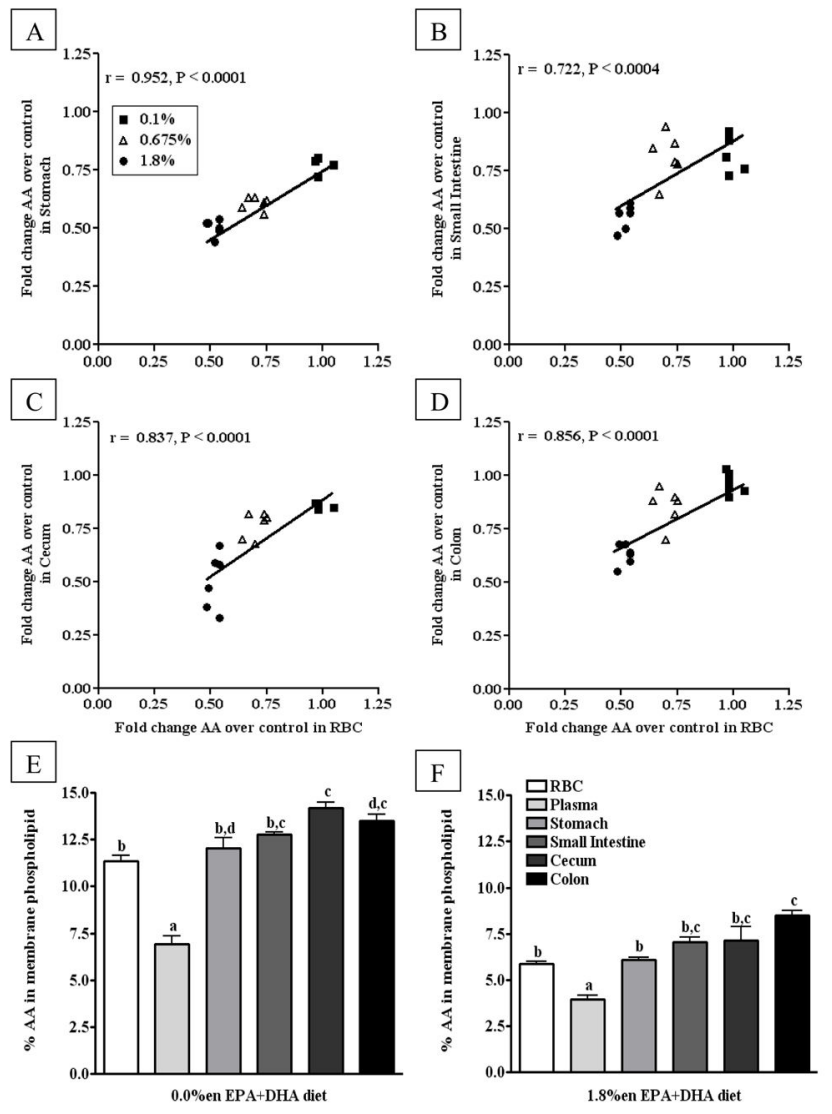

Figure 4. Comparison of AA content in phospholipid fatty acids from blood and gastrointestinal tissues from mice fed diets with increasing EPA+DHA

(A-D) Fold Change in AA content over the control group in phospholipid fatty acids from gastrointestinal tissues correlated to AA content in phospholipid fatty acids from red blood cells: (A) Stomach, (B) Small Intestine (C) Cecum, (D) Colon. Data from the tissues of mice fed experimental diets containing EPA+DHA-enriched fish oil were normalized to the control group to assess fold changes in AA content of phospholipids. A Pearson correlation was used to test linear correlation between gastrointestinal tissue AA fold change and RBC AA fold change $(\mathrm{n}=18)$. (E-F) The AA content from phospholipid fatty acids across blood and gastrointestinal tissues from mice fed either the control (E) or 1.8\%en EPA+DHA diet (F) $(n=6 /$ group). A one-way ANOVA and a Tukey's post-hoc were used to assess differences in AA content comparing all tissues to each other. Differing letters denote statistical significance at $P<0.05$. 


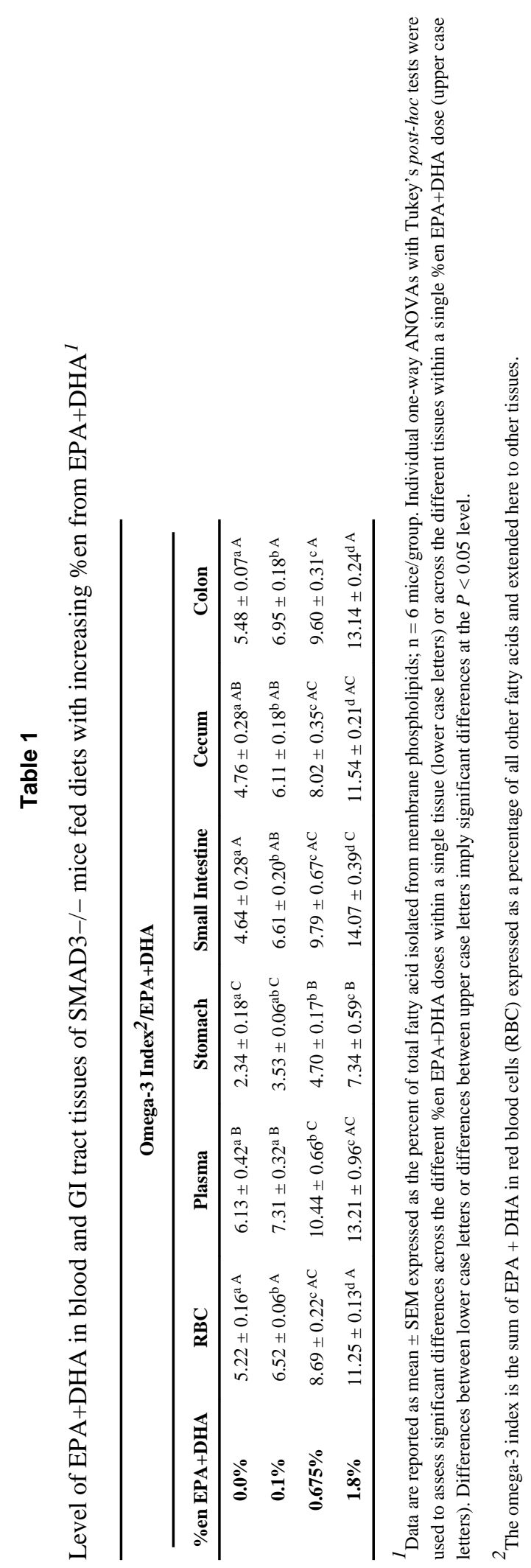

Prostaglandins Leukot Essent Fatty Acids. Author manuscript; available in PMC 2015 September 01. 
Table 2

Pearson Correlation Coefficients for n-3 or n-6 LCPUFAs between red blood cells and specified tissues in SMAD3-/- fed diets with increasing \%en from EPA+DHA

\begin{tabular}{ccccc}
\hline & \multicolumn{4}{c}{ Pearson Correlation Coefficients ${ }^{\mathbf{1}}$ against Red Blood Cell } \\
\cline { 2 - 5 } & EPA & DHA & AA & EPA+DHA \\
\hline Plasma & 0.912 & 0.752 & 0.894 & 0.920 \\
Stomach & 0.969 & 0.846 & 0.867 & 0.916 \\
Small Intestine & 0.963 & 0.947 & 0.811 & 0.967 \\
Cecum & 0.915 & 0.948 & 0.885 & 0.977 \\
Colon & 0.910 & 0.936 & 0.877 & 0.982 \\
\hline
\end{tabular}

${ }^{1}$ All Pearson correlation coefficients assessed the linear correlation between red blood cells vs. plasma or GI tissue for the fatty acids specified. All Pearson's $r$ coefficients were statistically significant at the $\mathrm{P}<0.0001$. 